\title{
Are There Really No Such Things as Theories?
}

\author{
Finnur Dellsén
}

[Penultimate draft of contribution to a symposium on Steven French's There Are No Such Things as Theories (Oxford: Oxford University Press, 2020))]

Philosophical discussions about scientific theories have largely centered on the conceptual question of how to define or analyze scientific theories and other similar representations, such as hypotheses and models. The two classic answers are the syntactic view, which holds that theories are sentences in an axiomatic system, and the semantic view, which holds that theories are collections of nonlinguistic models. In There Are No Such Things as Theories, Steven French, a champion of a version of the semantic view, takes a step back from this debate to focus on the ontological status of theories, asking: In what way, if any, do theories exist?

French's book is a tour de force through various extant and conceivable views of the ontological status of theories, e.g. that theories are abstract entities that are discovered in logical space, or that theories are fictions that serve as 'props' in scientific games of makebelief. Although French ultimately offers a different perspective on the ontological status of theories, he elaborates these extant views in ways that are clearly designed to provide us with the strongest possible versions thereof. Thus French's discussion is not only exceptionally charitable to his actual and potential opponents, but likely to advance his opponents' views in ways they had perhaps themselves not realized was possible.

As the title of his book indicates, French ends up defending what he describes as an 'eliminativist' view of theories. To a first approximation, this view holds that theories 
do not really exist. Instead, "all that really exists [are] the complex set of practices of the scientific community: the writing and dissemination of articles, the performance of experiments, the kinds of heuristic moves [by which theories are said to be 'discovered'], and so on" (191). The obvious problem with this claim is that we do seem to be able to talk coherently about theories in a way that we cannot do for obviously non-existent objects such as the largest prime number. As French puts it: “Obviously we don't want such elimination to force a revision of our language-we still want to be able to talk about theories and models" (186).

French's solution to this problem of reconciling eliminativism with meaningful theory-talk appeals to a version of truthmaker theory developed by Ross Cameron (2008b), in part with special reference to the ontological status of musical works (Cameron 2008a). On Cameron's view, a proposition of the form $<a$ exists $>^{1}$ can in some cases be made true by something other than $a$. In particular, the proposition $<$ theories exist $>$ can be made true by the practices of the relevant scientific community rather than by some abstract or fictional entities whose ontological status would arguably be problematic. In this way, says French, we avoid ontological commitment to theories while being able to talk about theories and even affirm their existence using ordinary English sentences such as 'theories exist'.

This is an attractive proposal insofar as it would allow us to simultaneously have our theory-cake and $e($ limin)at(e) it too. But does it work? Unless I am misunderstanding French's proposal, I see two main difficulties. The first concerns whether this Cameronian truthmaking maneuver really allows us to eliminate theories from our ontology, as

\footnotetext{
${ }^{1}$ I use angle brackets to denote propositions and single quotation marks to denote sentences.
} 
opposed to reducing them to something ontologically more innocuous. The problem is this: If the sentence 'theories exist' is true, then, by disquotation, theories exist. According to Cameronian truthmaker theory, the sentence 'theories exists', or indeed the corresponding proposition $<$ theories exist $>$, is not made true by the fact that theories exist. Nevertheless, that theories exist is still directly entailed by the truth of 'theories exist'. So even if it is not theories, or their existence, that makes 'theories exist' true, a Cameronian truthmaker theorist who accepts that 'theories exist' is true remains committed, in the sense of it being entailed by something they accept, to the existence of theories. This sounds a lot like ontological commitment to theories.

To be sure, Cameronian truthmaker theory has a ready-made response to this argument (see Cameron 2008, 6). The response is to adopt a definition of 'ontological commitment' that severs the link between accepting that $x$ s exist and ontological commitment to $x$ s. Specifically, the suggestion is that one is ontologically committed to $x \mathrm{~s}$ only if $x \mathrm{~s}$ are the truthmakers of sentences or propositions one accepts as true. This allows French to claim that he is 'ontologically committed' only to whatever it is that makes it true that theories exist - which, for French, are the various practices of scientific communities, dissemination of articles, performance of experiments, heuristic moves, etc. Note that this is a revision of the classic Quinean notion of ontological commitment according to which, roughly, one is ontologically committed to entities whose existence is entailed by sentences or propositions one accepts as true.

But how does this revision of the concept of 'ontological commitment' help us solve the philosophical puzzle about the ontological status of theories with which we began? Call it whatever you like, but when the propositions one accepts as true entail that something exists, that seems to be a type of commitment that is of some philosophical 
significance. In particular, if the claims you accept entail that theories exist while the claims I accept do not, then it seems that you are in some sense committed to the existence of theories while I am not. Even if you respond by insisting that the 'theories exist' is made true by practices rather than by some mysterious abstract or fictional entities, I can respond that your ontology still looks bloated from my point of view since you, implicitly or explicitly, accept that theories exist while I do not. At the very least, this puts pressure on the idea that French's suggestion really eliminates theories from his ontology. At most, it seems, it reduces theories to the practices that serve as truthmakers for theory-claims.

One potential response to this worry is that it ignores French's invocation of the language of 'Ontologese'. I have indeed avoided referring to this device - in part because French himself minimizes its importance $(184$, n. 8$)$, and in part because I fail to see how it helps to develop the truthmaking strategy. To explain, Ontologese is meant to be "the language of the fundamental level" (187) such that a sentence of the form ' $a$ exists' is true in Ontologese only if $a$ really does exist "at the fundamental level of the world" (184). The idea, then, is that our ontological commitments are revealed only by what is entailed by our claims in Ontologese as opposed to English. Since the Ontologese sentence 'theories exist' is false, says French, there is no ontological commitment to theories.

However, I fail to see how invoking Ontologese helps here. Let us start with the obvious: I do not speak Ontologese, and neither do you. If we did, then it clearly would not be another language separate from the actual languages that we do speak. So, in order for the two of us to even understand what our ontological commitments are, let alone make ontologically committing claims in this language, we would need a translation manual that tells us what the words or sentences of Ontologese mean in English or another language we actually understand. This translation manual would tell 
us, among other things, what the Ontologese terms 'theories' and 'exist' mean in English. But then why not simply use the English translation of the Ontologese terms to say what you wanted to say in English instead? The route through the artificial language of Ontologese seems unnecessary and superfluous. If, on the other hand, advocates Ontologese cannot provide us with translations of these terms, then it is unclear in what sense these commitments could be our commitments, since we could not even understand the sentences expressing them.

Indeed, there is a further, related difficulty. Note that whatever it is that the Ontologese terms 'theories' and 'exist' mean, it cannot be what the English terms 'theories' and 'exist' mean, since the sentence 'theories exist' is supposed to be true in English but false in Ontologese. At least one of the Ontologese terms 'theories' and 'exist' would be a false friend of the corresponding English terms, i.e. a word that that looks identical in two languages but differs in meaning between them. But then in what sense, if indeed any at all, is the fact that the Ontologese sentence 'theories exist' is false any kind of elimination of theories as we understand them? We speak English, not Ontologese, so insofar as Ontologese comes apart from English, it has no bearing on what we could possibly be interested in when we puzzle over the existence of theories. After all, our puzzle concerns the ontological status of what we refer to as theories, using our concept of existence.

For these reasons, I have doubts about French's proposed method for eliminating theories from our ontology, using Cameronian truthmaker theory and possibly the device of Ontologese. Rather, what French is offering seems to me to be an attempt at reducing theories to certain practices. 
On a more constructive note, let me suggest a different route for doing away with theories from our ontology with a more familiar method of ontological housekeeping. That method is Quine's good-old criterion of ontological commitment in its original 'desert landscapes' version that Quine himself preferred (Quine 1948). According to this method, we are to read our ontological commitments off the existence statements that are entailed by the statements we accept as true, although with the important caveat that we are allowed to paraphrase away any terms that seemingly refer to things to which we prefer not to be ontologically committed.

Admittedly, how exactly to achieve such paraphrasing-away is not obvious in any given case. For some things, e.g. for numbers, it is far from trivial (Field 1980). In the case of theories, one would have to find a way to paraphrase any sentence one accepts about theories in terms of something else, e.g. scientists' mental states or, as French would no doubt prefer, their practices. Provided that this type of paraphrasing-away can be achieved, a theory-free ontology would not force us to abandon theory-talk, for speaking in terms of theories would entail no ontological commitment to anything over and above the things in the paraphrased sentences, e.g. mental states or practices. Again, this will no doubt be a difficult task, but it seems to be an option worth exploring for those who would like to entirely eliminate theories from their ontology. Given that French adopts Cameron's notion of ontological commitment, which is an alternative to Quine's, one strongly suspects that French would not be happy with this Quinean project. But it would have been interesting to know on what grounds French prefers the Cameronian approach to the classic Quinean one.

I turn now to a second aspect of French's eliminativist proposal, viz. the idea that the truthmakers for claims about theories are the practices of a scientific community. 
Presumably, most or even all scientists could be mistaken about some aspect of a theory $T$, e.g. whether $T$ entails some prediction $P$. This, in turn, could be reflected in scientists' practices, for example in what they write and disseminate about $T$, and how they experiment on $T$. For example, scientists might take a refutation of $P$ to conclusively falsify $T$, even if $T$ as it is written down in articles, textbooks, etc. in fact does not entail $P$ at all. But if a theory is entirely made true by a set of practices, including the practice of taking a refutation of $P$ to falsify $T$, then how can we make sense of the claim that scientists are mistaken in taking $T$ to entail $P$ ? Would not the (intuitively mistaken) practice of taking a refutation of $P$ to falsify $T$ simply make it true that $T$ entails $P$ ?

Suppose, for instance, that physicists mistakenly thought that the theory of special relativity as it was actually formulated by Einstein in 1905 entails that the speed of light, $c$, is the same in any medium. Moreover, suppose that this would be reflected in physicists' practices, e.g. by their taking observed differences in $c$ in water and air as a conclusive falsification of this theory. Everything else about Einstein's theory of special relativity, including how the theory itself is written down in published articles, textbooks, and correspondence, is exactly the same as in the actual world. French's proposal seems to entail that, in this hypothetical scenario, Einstein's theory of special relativity would be a different theory from what it is in the actual world, since the practices which make it what it is would be significantly different. But then it seems to follow that Einstein's theory of special relativity, grounded as it is in these mistaken practices, would entail that $c$ is the same in any medium. More generally, there would seem to be some sets of possible practices (not necessarily those described here) so as to make it true that Einstein's theory of special relativity entails that $c$ is the same in any medium. 
To be clear, the worry here is not simply that the theory that happens to be called 'the special theory of relativity' would be a different theory if the relevant practices were different. Rather, the worry is that French's proposal seems to eliminate the possibility that some of the theory-related practices of a given community are simply mistaken, such as the intuitively-mistaken practice of taking observed differences in $c$ to refute the special theory of relativity as Einstein formulated it in 1905. As long as most or all scientists in a community adopt the relevant set of practices, the corresponding claims about the theory automatically become true, so one cannot criticize or reject their claims on any basis other than the question-begging one that we do things differently in our community. In general, once we ground the truth of theory-claims in any aspect of communal thought or behavior, it seems to me that we lose the ability to criticize the relevant aspect of communal thought or behavior in a non-question-begging way.

To my mind, this points to a key difficulty for any attempt to ground the truth of theory-claims in scientists' practices, or their mental states, for that matter. Once a theory has been formulated, e.g. as Einstein did with special relativity in 1905, there are certain objective truths about the theory, e.g. regarding what it does and does not entail. If the practices of scientists at a given time do not match these truths, then these practices are objectively mistaken: Einstein's theory of special relativity is not refuted by observed differences in $c$ in different media, no matter what the practices of the scientific community are at any given time. But how can we make sense of these types of objective mistakes about theories if theory-claims are entirely made true by the very practices that would be mistaken?

\section{Acknowledgements}


Many thanks to James Norton for very helpful comments on, and discussions about, an earlier draft.

\section{References}

Cameron, R. (2008a). There Are No Things That Are Musical Works. British Journal of Aesthetics 48: 295-314.

Cameron, R. (2008b). Truthmakers and Ontological Commitment. Philosophical Studies 140: 1-18.

Field, H. 1980. Science Without Numbers: A Defense of Nominalism. Oxford: Blackwell.

Quine, W.v.O. (1948). On What There Is. The Review of Metaphysics 5: 21-38. 